\title{
Article
}

\section{Some Integral Inequalities Involving Metrics}

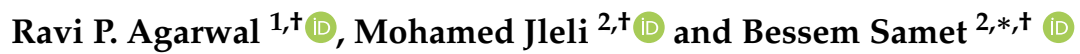 \\ 1 Department of Mathematics, Texas A \& M University-Kingsville, Kingsville, TX 78363, USA; \\ Ravi.Agarwal@tamuk.edu \\ 2 Department of Mathematics, College of Science, King Saud University, P.O. Box 2455, \\ Riyadh 11451, Saudi Arabia; jleli@ksu.edu.sa \\ * Correspondence: bsamet@ksu.edu.sa \\ + These authors contributed equally to this work.
}

check for

updates

Citation: Agarwal, R.P.; Jleli, M.; Samet, B. Some Integral Inequalities Involving Metrics. Entropy 2021, 23, 871. https://doi.org/10.3390/ e23070871

Academic Editor: António M. Lopes

Received: 29 June 2021

Accepted: 4 July 2021

Published: 8 July 2021

Publisher's Note: MDPI stays neutral with regard to jurisdictional claims in published maps and institutional affiliations.
Abstract: In this work, we establish some integral inequalities involving metrics. Moreover, some applications to partial metric spaces are given. Our results are extension of previous obtained metric inequalities in the discrete case.

Keywords: integral inequalities; metric; partial metric; sub-additive; convex; log-convex; $\sigma$-Lipschitzian

\section{Introduction}

Metric inequalities provide powerful tools for the investigation of several problems from different branches of mathematics and sciences. In particular, from entropy and information theory (see e.g., [1-3]), fixed point theory (see e.g., [4-8]), geometry (see e.g., [9-11]) and telecommunication networks (see e.g., [12,13]).

In [14], Dragomir and Gosa established a polygonal type inequality and provided some applications to normed linear spaces and inner product spaces. We recall below the main result obtained in [14]. Let $\mathcal{X}$ be a nonempty set and $\rho: \mathcal{X} \times \mathcal{X} \rightarrow[0,+\infty)$ be a metric on $\mathcal{X}$ (see [15]), that is, for all $a, b, c \in \mathcal{X}$,

- $\rho(a, b)=0$ if and only if $a=b$;

- $\rho(a, b)=\rho(b, a)$;

- $\rho(a, b) \leq \rho(a, c)+\rho(c, b)$.

Let $n \geq 2$ be a natural number, $\left\{x_{i}\right\}_{i=1}^{n} \subset \mathcal{X}$, and $\left\{\mu_{i}\right\}_{i=1}^{n} \subset[0,+\infty)$ with $\mu_{1}+$ $\cdots \mu_{n}=1$. Then

$$
\sum_{1 \leq i<j \leq n} \mu_{i} \mu_{j} \rho\left(x_{i}, x_{j}\right) \leq \inf _{x \in \mathcal{X}} \sum_{i=1}^{n} \mu_{i} \rho\left(x_{i}, x\right) .
$$

It was shown also that (1) is sharp in the following sense: there exists $n \geq 2$ and $\left\{\mu_{i}\right\}_{i=1}^{n} \subset[0,+\infty)$ with $\mu_{1}+\cdots \mu_{n}=1$ such that, if

$$
\sum_{1 \leq i<j \leq n} \mu_{i} \mu_{j} \rho\left(x_{i}, x_{j}\right) \leq c \inf _{x \in \mathcal{X}} \sum_{i=1}^{n} \mu_{i} \rho\left(x_{i}, x\right)
$$

for some $c>0$, then $c \geq 1$. Inequality (1) can be interpreted as follows: If $\mathcal{P}$ is a polygon having $q$ vertices and $M$ is a point in the space, then the sum of all edges and diagonals of $\mathcal{P}$ is less than $q$-times the sum of the distances from $M$ to the vertices of $\mathcal{P}$. 
Recently, inequality (1) has been extended by some authors. In [16], Karapinar and Noorwali derived a $b$-metric version of (1). In [17], Aydi and Samet proved (under the above assumptions) that

$$
\begin{aligned}
& \frac{1}{2} \sum_{1 \leq i<j \leq n} \mu_{i} \mu_{j}\left[\rho\left(x_{i}, x_{j}\right)\right]^{m} \\
& \leq \inf _{x \in \mathcal{X}}\left[2 \sum_{i=1}^{n} \mu_{i}\left[\rho\left(x_{i}, x\right)\right]^{m}+\sum_{k=1}^{m-1}\left(\begin{array}{c}
m \\
k
\end{array}\right)\left(\sum_{i=1}^{n} \mu_{i}\left[\rho\left(x_{i}, x\right)\right]^{k}\right)\left(\sum_{i=1}^{n} \mu_{i}\left[\rho\left(x_{i}, x\right)\right]^{m-k}\right)\right],
\end{aligned}
$$

where $m \geq 1$ is a natural number. In [18] (see also [19]) Dragomir improved inequality (1) by proving that for all $\alpha>0$,

$$
\sum_{1 \leq i<j \leq n} \mu_{i} \mu_{j}\left[\rho\left(x_{i}, x_{j}\right)\right]^{\alpha} \leq a_{\alpha} \inf _{x \in \mathcal{X}} \sum_{i=1}^{n} \mu_{i}\left(1-\mu_{i}\right)\left[\rho\left(x_{i}, x\right)\right]^{\alpha},
$$

where

$$
a_{\alpha}=\left\{\begin{array}{lll}
2^{\alpha-1} & \text { if } \quad \alpha \geq 1 \\
1 & \text { if } & 0<\alpha<1
\end{array}\right.
$$

In this paper, our goal is to derive continuous versions of inequality (2). In the next section, we recall some basic definitions. In Section 3, we present and prove our obtained results. Finally, in Section 4, some applications to partial metric spaces are provided.

\section{Some Definitions}

Let $\omega:[0,+\infty) \rightarrow[0,+\infty)$ be a given function. We say that $\omega$ is sub-additive, if

$$
\omega(y+z) \leq \omega(y)+\omega(z), \quad \text { for all } y, z \geq 0 .
$$

Some examples of sub-additive functions are given below:

- $\omega_{\alpha}(y)=y^{\alpha}, y \geq 0,0 \leq \alpha \leq 1$.

- $\omega(y)=|\sin y|, y \geq 0$.

- $\omega(y)=\arctan y, y \geq 0$.

- $\omega(y)=\inf \{y, 1\}, y \geq 0$.

- $\omega(y)=\sqrt{1+y^{2}}, y \geq 0$.

- $\omega(y)=\exp (-y), y \geq 0$.

We say that $\omega$ is convex, if

$$
\omega(\phi y+(1-\phi) z) \leq \phi \omega(y)+(1-\phi) \omega(z),
$$

for all $0<\phi<1$ and $y, z \geq 0$.

We say that $\omega$ is log-convex, if

$$
\omega(\phi y+(1-\phi) z) \leq[\omega(y)]^{\phi}[\omega(z)]^{1-\phi},
$$

for all $0<\phi<1$ and $y, z \geq 0$. Notice that, if $\omega$ is log-convex, then it is convex, but the converse is not true in general (see [20]).

We say that $\omega$ is $\sigma$-Lipschitzian, $\sigma>0$, if

$$
|\omega(y)-\omega(z)| \leq \sigma|y-z|, \quad \text { for all } y, z \geq 0 .
$$

\section{Results and Proofs}

Let $(\mathcal{X}, \rho)$ be a metric space. Let $x:[0, A] \rightarrow \mathcal{X}, A>0$, be a continuous mapping. Let $\mu:[0, A] \rightarrow[0,+\infty)$ be a function satisfying the following conditions:

(H1) $\mu$ is continuous. 
(H2) $\int_{0}^{A} \mu(s) d s=1$.

3.1. The Case: $\omega$ Is Sub-Additive

Theorem 1. Let $\omega:[0,+\infty) \rightarrow[0,+\infty)$ be a continuous, nondecreasing, and sub-additive function. Then

$$
\int_{0}^{A} \mu(t)\left(\int_{0}^{t} \mu(s) \omega(\rho(x(t), x(s))) d s\right) d t \leq \inf _{u \in \mathcal{X}} \int_{0}^{A} \mu(s) \omega(\rho(x(s), u)) d s .
$$

Proof. Let $u \in \mathcal{X}$ be fixed. Then, for every $t, s \in[0, A]$,

$$
\rho(x(t), x(s)) \leq \rho(x(t), u)+\rho(u, x(s)) .
$$

Since $\omega$ is nondecreasing, the above inequality leads to

$$
\omega(\rho(x(t), x(s))) \leq \omega(\rho(x(t), u)+\rho(u, x(s))) .
$$

Due to the sub-additivity of $\omega$, it holds that

$$
\omega(\rho(x(t), x(s))) \leq \omega(\rho(x(t), u))+\omega(\rho(u, x(s))) .
$$

Multiplying the above inequality by $\mu(t) \mu(s)$ (notice that $\mu \geq 0$ ) and integrating over $[0, A] \times[0, A]$, we obtain

$$
\begin{aligned}
\int_{0}^{A} \int_{0}^{A} \mu(t) \mu(s) \omega(\rho(x(t), x(s))) d t d s \leq & \int_{0}^{A} \int_{0}^{A} \mu(t) \mu(s) \omega(\rho(x(t), u)) d t d s \\
& +\int_{0}^{A} \int_{0}^{A} \mu(t) \mu(s) \omega(\rho(u, x(s)) d t d s .
\end{aligned}
$$

On the other hand, by (H2), we have

$$
\begin{aligned}
\int_{0}^{A} \int_{0}^{A} \mu(t) \mu(s) \omega(\rho(x(t), u)) d t d s & =\left(\int_{0}^{A} \mu(s) d s\right)\left(\int_{0}^{A} \mu(t) \omega(\rho(x(t), u)) d t\right) \\
& =\int_{0}^{A} \mu(t) \omega(\rho(x(t), u)) d t .
\end{aligned}
$$

Similarly,

$$
\int_{0}^{A} \int_{0}^{A} \mu(t) \mu(s) \omega(\rho(u, x(s))) d t d s=\int_{0}^{A} \mu(s) \omega(\rho(u, x(s))) d s .
$$

Combining the above inequalities, we obtain

$$
\begin{aligned}
& \int_{0}^{A} \int_{0}^{A} \mu(t) \mu(s) \omega(\rho(x(t), u)) d t d s+\int_{0}^{A} \int_{0}^{A} \mu(t) \mu(s) \omega(\rho(u, x(s))) d t d s \\
& =2 \int_{0}^{A} \mu(s) \omega(\rho(u, x(s))) d s .
\end{aligned}
$$

Moreover, we have

$$
\begin{aligned}
\int_{0}^{A} \int_{0}^{A} \mu(t) \mu(s) \omega(\rho(x(t), x(s))) d t d s= & \int_{0}^{A} \mu(t)\left(\int_{0}^{t} \mu(s) \omega(\rho(x(t), x(s))) d s\right) d t \\
& +\int_{0}^{A} \mu(t)\left(\int_{t}^{A} \mu(s) \omega(\rho(x(t), x(s))) d s\right) d t .
\end{aligned}
$$


On the other hand, using Fubini's theorem and the symmetry of $\rho$, we obtain

$$
\int_{0}^{A} \mu(t)\left(\int_{t}^{A} \mu(s) \omega(\rho(x(t), x(s))) d s\right) d t=\int_{0}^{A} \mu(t)\left(\int_{0}^{t} \mu(s) \omega(\rho(x(t), x(s))) d s\right) d t .
$$

Therefore, by (7) and (8), we deduce that

$$
\int_{0}^{A} \int_{0}^{A} \mu(t) \mu(s) \omega(\rho(x(t), x(s))) d t d s=2 \int_{0}^{A} \mu(t)\left(\int_{0}^{t} \mu(s) \omega(\rho(x(t), x(s))) d s\right) d t .
$$

Finally, (4) follows from (5), (6) and (9).

Next, we study some special cases of $\omega$. In the case $\omega(y)=y^{\alpha}, 0<\alpha \leq 1$, we deduce from Theorem 1 the

Corollary 1. Let $0<\alpha \leq 1$. Then

$$
\int_{0}^{A} \mu(t)\left(\int_{0}^{t} \mu(s)[\rho(x(t), x(s))]^{\alpha} d s\right) d t \leq \inf _{u \in \mathcal{X}} \int_{0}^{A} \mu(s)[\rho(x(s), u)]^{\alpha} d s .
$$

In the special case when $\omega(y)=\sqrt{\alpha+y^{2}}, \alpha>0$, we deduce from Theorem 1 the

Corollary 2. Let $\alpha>0$. Then

$$
\int_{0}^{A} \mu(t)\left(\int_{0}^{t} \mu(s) \sqrt{\alpha+[\rho(x(t), x(s))]^{2}} d s\right) d t \leq \inf _{u \in \mathcal{X}} \int_{0}^{A} \mu(s) \sqrt{\alpha+[\rho(x(s), u)]^{2}} d s .
$$

In the case when $\omega(y)=\arctan y$, we deduce from Theorem 1 the

Corollary 3. The following inequality holds:

$$
\int_{0}^{A} \mu(t)\left(\int_{0}^{t} \mu(s) \arctan (\rho(x(t), x(s))) d s\right) d t \leq \inf _{u \in \mathcal{X}} \int_{0}^{A} \mu(s) \arctan (\rho(x(s), u)) d s .
$$

3.2. The Case: $\omega$ Is Convex

Theorem 2. Let $\omega:[0,+\infty) \rightarrow[0,+\infty)$ be a nondecreasing and convex function. Then

$$
\int_{0}^{A} \mu(t)\left(\int_{0}^{t} \mu(s) \omega\left(\frac{\rho(x(t), x(s))}{2}\right) d s\right) d t \leq \frac{1}{2} \inf _{u \in \mathcal{X}} \int_{0}^{A} \mu(s) \omega(\rho(x(s), u)) d s .
$$

Proof. Fix $u \in \mathcal{X}$. Since $\omega$ is nondecreasing, we have

$$
\omega\left(\frac{\rho(x(t), x(s))}{2}\right) \leq \omega\left(\frac{\rho(x(t), u)+\rho(u, x(s))}{2}\right) .
$$

Due to the convexity of $\omega$, the above inequality leads to

$$
\omega\left(\frac{\rho(x(t), x(s))}{2}\right) \leq \frac{1}{2}[\omega(\rho(x(t), u))+\omega(\rho(u, x(s)))] .
$$

Multiplying the above inequality by $\mu(t) \mu(s)$ and integrating over $[0, A] \times[0, A]$, we obtain

$$
\begin{aligned}
\int_{0}^{A} \int_{0}^{A} \mu(t) \mu(s) \omega\left(\frac{\rho(x(t), x(s))}{2}\right) d t d s \leq & \frac{1}{2} \int_{0}^{A} \int_{0}^{A} \mu(t) \mu(s) \omega(\rho(x(t), u)) d t d s \\
& +\frac{1}{2} \int_{0}^{A} \int_{0}^{A} \mu(t) \mu(s) \omega(\rho(u, x(s)) d t d s
\end{aligned}
$$


Proceeding as in the proof of Theorem 1, we obtain

$$
\int_{0}^{A} \int_{0}^{A} \mu(t) \mu(s) \omega\left(\frac{\rho(x(t), x(s))}{2}\right) d t d s=2 \int_{0}^{A} \mu(t)\left(\int_{0}^{t} \mu(s) \omega\left(\frac{\rho(x(t), x(s))}{2}\right) d s\right) d t
$$

and

$$
\begin{aligned}
& \frac{1}{2} \int_{0}^{A} \int_{0}^{A} \mu(t) \mu(s) \omega(\rho(x(t), u)) d t d s+\frac{1}{2} \int_{0}^{A} \int_{0}^{A} \mu(t) \mu(s) \omega(\rho(u, x(s)) d t d s \\
& =\int_{0}^{A} \mu(s) \omega(\rho(x(s), u)) d s .
\end{aligned}
$$

Finally, combining (11), (12), and (13), (10) follows.

In the special case when $\omega(y)=y^{\alpha}, \alpha>1$, by Theorem 2 , we deduce the following result.

Corollary 4. Let $\alpha>1$. Then

$$
\int_{0}^{A} \mu(t)\left(\int_{0}^{t} \mu(s)[\rho(x(t), x(s))]^{\alpha} d s\right) d t \leq 2^{\alpha-1} \inf _{u \in \mathcal{X}} \int_{0}^{A} \mu(s)[\rho(x(s), u)]^{\alpha} d s .
$$

3.3. The Case: $\omega$ Is log-Convex

Theorem 3. Let $\omega:[0,+\infty) \rightarrow[0,+\infty)$ be a nondecreasing and log-convex function. Then

$$
\int_{0}^{A} \mu(t)\left(\int_{0}^{t} \mu(s) \omega\left(\frac{\rho(x(t), x(s))}{2}\right) d s\right) d t \leq \frac{1}{2} \inf _{u \in \mathcal{X}}\left(\int_{0}^{A} \mu(s) \sqrt{\omega(\rho(x(s), u))} d s\right)^{2} .
$$

Proof. Fix $u \in \mathcal{X}$. Since $\omega$ is nondecreasing, we have

$$
\omega\left(\frac{\rho(x(t), x(s))}{2}\right) \leq \omega\left(\frac{\rho(x(t), u)+\rho(u, x(s))}{2}\right) .
$$

Due to the log-convexity of $\omega$, the above inequality leads to

$$
\omega\left(\frac{\rho(x(t), x(s))}{2}\right) \leq \sqrt{\omega(\rho(x(t), u))} \sqrt{\omega(\rho(u, x(s)))} .
$$

Multiplying the above inequality by $\mu(t) \mu(s)$ and integrating over $[0, A] \times[0, A]$, we obtain

$$
\begin{aligned}
& \int_{0}^{A} \int_{0}^{A} \mu(t) \mu(s) \omega\left(\frac{\rho(x(t), x(s))}{2}\right) d t d s \\
& \leq \int_{0}^{A} \int_{0}^{A} \mu(t) \mu(s) \sqrt{\omega(\rho(x(t), u))} \sqrt{\omega(\rho(u, x(s)))} d t d s .
\end{aligned}
$$

On the other hand,

$\int_{0}^{A} \int_{0}^{A} \mu(t) \mu(s) \sqrt{\omega(\rho(x(t), u))} \sqrt{\omega(\rho(u, x(s)))} d t d s=\left(\int_{0}^{A} \mu(s) \sqrt{\omega(\rho(x(s), u))} d s\right)^{2}$.

Hence, using (12), (15) and (16), (14) follows.

Consider the special case $\omega(y)=\exp \left(y^{\alpha}\right), \alpha \geq 1$. By Theorem 3 , we obtain the

Corollary 5. Let $\alpha \geq 1$. Then

$$
\int_{0}^{A} \mu(t)\left(\int_{0}^{t} \mu(s) \exp \left[\left(\frac{\rho(x(t), x(s))}{2}\right)^{\alpha}\right] d s\right) d t \leq \frac{1}{2} \inf _{u \in \mathcal{X}}\left(\int_{0}^{A} \mu(s) \sqrt{\exp \left[(\rho(x(s), u))^{\alpha}\right]} d s\right)^{2} .
$$




\subsection{The Case: $\omega$ Is $\sigma$-Lipschitzian}

Theorem 4. Let $\omega:[0,+\infty) \rightarrow[0,+\infty)$ be a nondecreasing and $\sigma$-Lipschitzian function, $\sigma>0$. Then

$$
\int_{0}^{A} \mu(t)\left(\int_{0}^{t} \mu(s) \omega(\rho(x(t), x(s))) d s\right) d t \leq \sigma \inf _{u \in \mathcal{X}}\left[\int_{0}^{A} \mu(s) \omega(\rho(x(s), u)) d s\right]+\frac{\omega(0)}{2} .
$$

Proof. Let $u \in \mathcal{X}$ be fixed. Then, for every $t, s \in[0, A]$,

$$
\rho(x(t), x(s)) \leq \rho(x(t), u)+\rho(u, x(s)) .
$$

Since $\omega$ is nondecreasing, the above inequality leads to

$$
\omega(\rho(x(t), x(s))) \leq \omega(\rho(x(t), u)+\rho(u, x(s))) .
$$

On the other hand, since $\omega$ is $\sigma$-Lipschitzian, we have

$$
\begin{aligned}
\omega(\rho(x(t), u)+\rho(u, x(s))) & =[\omega(\rho(x(t), u)+\rho(u, x(s)))-\omega(0)]+\omega(0) \\
& \leq \sigma[\rho(x(t), u)+\rho(u, x(s))]+\omega(0) .
\end{aligned}
$$

Hence, it holds that

$$
\omega(\rho(x(t), x(s))) \leq \sigma[\rho(x(t), u)+\rho(u, x(s))]+\omega(0) .
$$

Multiplying the above inequality by $\mu(t) \mu(s)$ and integrating over $[0, A] \times[0, A]$, we obtain

$$
\begin{aligned}
& \int_{0}^{A} \int_{0}^{A} \mu(t) \mu(s) \omega(\rho(x(t), x(s))) d t d s \\
& \leq \sigma \int_{0}^{A} \int_{0}^{A} \mu(t) \mu(s) \omega(\rho(x(t), u)) d t d s+\sigma \int_{0}^{A} \int_{0}^{A} \mu(t) \mu(s) \omega(\rho(u, x(s)) d t d s \\
& +\omega(0) \int_{0}^{A} \int_{0}^{A} \mu(t) \mu(s) d t d s \\
& =\sigma \int_{0}^{A} \int_{0}^{A} \mu(t) \mu(s) \omega(\rho(x(t), u)) d t d s+\sigma \int_{0}^{A} \int_{0}^{A} \mu(t) \mu(s) \omega(\rho(u, x(s)) d t d s+\omega(0) .
\end{aligned}
$$

Next, using (6), (9) and (18), we deduce that

$$
2 \int_{0}^{A} \mu(t)\left(\int_{0}^{t} \mu(s) \omega(\rho(x(t), x(s))) d s\right) d t \leq 2 \sigma \int_{0}^{A} \mu(s) \omega(\rho(u, x(s))) d s+\omega(0),
$$

which yields (17).

\subsection{The Case: $\mu \equiv A^{-1}$}

Consider now the case when

$$
\mu(t)=A^{-1}, \quad \text { for all } t \in[0, A] .
$$

Then by Theorems 1-4, we deduce the following inequalities.

Corollary 6. Let $\omega:[0,+\infty) \rightarrow[0,+\infty)$ be a continuous, nondecreasing, and sub-additive function. Then

$$
\int_{0}^{A}\left(\int_{0}^{t} \omega(\rho(x(t), x(s))) d s\right) d t \leq A \inf _{u \in \mathcal{X}} \int_{0}^{A} \omega(\rho(x(s), u)) d s .
$$


Corollary 7. Let $\omega:[0,+\infty) \rightarrow[0,+\infty)$ be a nondecreasing and convex function. Then

$$
\int_{0}^{A}\left(\int_{0}^{t} \omega\left(\frac{\rho(x(t), x(s))}{2}\right) d s\right) d t \leq \frac{A}{2} \inf _{u \in \mathcal{X}} \int_{0}^{A} \omega(\rho(x(s), u)) d s .
$$

Corollary 8. Let $\alpha>0$. Then

$$
\int_{0}^{A}\left(\int_{0}^{t}[\rho(x(t), x(s))]^{\alpha} d s\right) d t \leq a_{\alpha} A \inf _{u \in \mathcal{X}} \int_{0}^{A}[\rho(x(s), u)]^{\alpha} d s,
$$

where $a_{\alpha}$ is given by (3).

Corollary 9. Let $\omega:[0,+\infty) \rightarrow[0,+\infty)$ be a nondecreasing and log-convex function. Then

$$
\int_{0}^{A}\left(\int_{0}^{t} \omega\left(\frac{\rho(x(t), x(s))}{2}\right) d s\right) d t \leq \frac{1}{2} \inf _{u \in \mathcal{X}}\left(\int_{0}^{A} \sqrt{\omega(\rho(x(s), u))} d s\right)^{2} .
$$

Corollary 10. Let $\omega:[0,+\infty) \rightarrow[0,+\infty)$ be a nondecreasing and $\sigma$-Lipschitzian function, $\sigma>0$. Then

$$
\int_{0}^{A}\left(\int_{0}^{t} \omega(\rho(x(t), x(s))) d s\right) d t \leq \sigma A \inf _{u \in \mathcal{X}}\left[\int_{0}^{A} \omega(\rho(x(s), u)) d s\right]+\frac{A^{2} \omega(0)}{2} .
$$

\section{Applications to Partial Metrics}

Let $\mathcal{X}$ be a nonempty set and $\xi: \mathcal{X} \times \mathcal{X} \rightarrow[0,+\infty)$ be a partial metric on $\mathcal{X}$ (see e.g., [21]), i.e., for all $x, y, z \in \mathcal{X}$,

- $\xi(x, y)=\xi(x, x)=\xi(y, y)=0$ if and only if $x=y$;

- $\xi(x, x) \leq \xi(x, y)$;

- $\xi(x, y)=\xi(y, x)$;

- $\xi(x, y) \leq \xi(x, z)+\xi(z, y)-\xi(z, z)$.

Let $x:[0, A] \rightarrow \mathcal{X}, A>0$, be a continuous mapping. Let $\mu:[0, A] \rightarrow[0,+\infty)$ be a function satisfying (H1) and (H2).

Corollary 11. Let $\omega:[0,+\infty) \rightarrow[0,+\infty)$ be a continuous, nondecreasing, and sub-additive function. Then

$$
\begin{aligned}
& \int_{0}^{A} \mu(t)\left(\int_{0}^{t} \mu(s) \omega(2 \xi(x(t), x(s))-\xi(x(t), x(t))-\xi(x(s), x(s))) d s\right) d t \\
& \leq \inf _{u \in \mathcal{X}} \int_{0}^{A} \mu(s) \omega(2 \xi(x(s), u)-\xi(x(s), x(s))-\xi(u, u)) d s .
\end{aligned}
$$

Proof. Observe that the mapping $\rho: \mathcal{X} \times \mathcal{X} \rightarrow[0,+\infty)$ defined by

$$
\rho(u, v)=2 \xi(u, v)-\xi(u, u)-\xi(v, v), \quad(u, v) \in \mathcal{X} \times \mathcal{X},
$$

is a metric on $\mathcal{X}$. Hence, applying Theorem 1 with $\rho$ defined as above, (19) follows.

Similarly, by Theorems $2-4$, we obtain the following inequalities.

Corollary 12. Let $\omega:[0,+\infty) \rightarrow[0,+\infty)$ be a nondecreasing and convex function. Then

$$
\begin{aligned}
& \int_{0}^{A} \mu(t)\left(\int_{0}^{t} \mu(s) \omega\left(\frac{2 \xi(x(t), x(s))-\xi(x(t), x(t))-\xi(x(s), x(s))}{2}\right) d s\right) d t \\
& \leq \frac{1}{2} \inf _{u \in \mathcal{X}} \int_{0}^{A} \mu(s) \omega(2 \xi(x(s), u)-\xi(x(s), x(s))-\xi(u, u)) d s .
\end{aligned}
$$


Corollary 13. Let $\omega:[0,+\infty) \rightarrow[0,+\infty)$ be a nondecreasing and log-convex function. Then

$$
\begin{aligned}
& \int_{0}^{A} \mu(t)\left(\int_{0}^{t} \mu(s) \omega\left(\frac{2 \xi(x(t), x(s))-\xi(x(t), x(t))-\xi(x(s), x(s))}{2}\right) d s\right) d t \\
& \leq \frac{1}{2} \inf _{u \in \mathcal{X}}\left(\int_{0}^{A} \mu(s) \sqrt{\omega(2 \xi(x(s), u)-\xi(x(s), x(s))-\xi(u, u))} d s\right)^{2} .
\end{aligned}
$$

Corollary 14. Let $\omega:[0,+\infty) \rightarrow[0,+\infty)$ be a nondecreasing and $\sigma$-Lipschitzian function, $\sigma>0$. Then

$$
\begin{aligned}
& \int_{0}^{A} \mu(t)\left(\int_{0}^{t} \mu(s) \omega(2 \xi(x(t), x(s))-\xi(x(t), x(t))-\xi(x(s), x(s))) d s\right) d t \\
& \leq \sigma \inf _{u \in \mathcal{X}}\left[\int_{0}^{A} \mu(s) \omega(2 \xi(x(s), u)-\xi(x(s), x(s))-\xi(u, u)) d s\right]+\frac{\omega(0)}{2} .
\end{aligned}
$$

\section{Conclusions}

Metric inequalities provide powerful tools for the study of several problems from different branches of mathematics and sciences. New integral inequalities involving metrics, sub-additive, convex, log-convex, and $\sigma$-Lipschitzian functions are established in this work, and some applications to partial metric spaces are provided. The obtained results are continuous versions of some discrete metric inequalities obtained in $[14,18,19]$.

Author Contributions: All authors contribute equally to this paper. All authors have read and agreed to the published version of the manuscript.

Funding: The third author is supported by Researchers Supporting Project number (RSP-2021/4), King Saud University, Riyadh, Saudi Arabia.

Institutional Review Board Statement: Not applicable.

Informed Consent Statement: Not applicable.

Data Availability Statement: Not applicable.

Conflicts of Interest: The authors declare no conflict of interest.

\section{References}

1. Cichocki, A.; Cruces, S.; Amari, S.I. Log-Determinant Divergences Revisited: Alpha-Beta and Gamma Log-Det Divergences. Entropy 2015, 17, 2988-3034. [CrossRef]

2. Liu, S.; Lu, M.; Liu, G.; Pan, Z. A Novel Distance Metric: Generalized Relative Entropy. Entropy 2017, 19, 269. [CrossRef]

3. Reeb, D.; Kastoryano, M.J.; Wolf, M.M. Hilbert's projective metric in quantum information theory. J. Math. Phys. $2011,52,082201$. [CrossRef]

4. Ansari, A.H.; Kumam, P.; Samet, B. A fixed point problem with constraint inequalities via an implicit contraction. J. Fixed Point Theory Appl. 2017, 19, 1145-1163 [CrossRef]

5. Jleli, M.; Samet, B. A fixed point problem under two constraint inequalities. Fixed Point Theory Appl. 2016, 18, 1-14. [CrossRef]

6. Mitrovic, Z.D.; Aydi, H.; Noorani, M.S.; Qawaqneh, H. The weight inequalities on Reich type theorem in b-metric spaces. J. Math. Comput. Sci. 2019, 19, 51-57. [CrossRef]

7. Simić, S.; Radenović, S. A functional inequality. J. Math. Anal. Appl. 1996, 117, 489-494. [CrossRef]

8. Suzuki, T. Basic inequality on a b-metric space and its applications. J. Inequal. Appl. 2017, 2017, 256. [CrossRef] [PubMed]

9. Gromov, M. Metric inequalities with scalar curvature. Geom. Funct. Anal. 2018, 28, 645-726. [CrossRef]

10. Lángi, Z. On the perimeters of simple polygons contained in a disk. Monatsh. Math. 2011, 162, 61-67. [CrossRef]

11. Witsenhausen, H.S. Metric inequalities and the zonoid problem. Proc. Am. Math. Soc. 1973, 40, 517-520. [CrossRef]

12. Mattia, S. Solving survivable two-layer network design problems by metric inequalities. Comput. Optim. Appl. 2012, 51, 809-834. [CrossRef]

13. Mattia, S. The robust network loading problem with dynamic routing. Comp. Opt. Appl. 2013, 54, 619-643. [CrossRef]

14. Dragomir, S.S.; Gosa, A.C. An inequality in metric spaces. J. Indones. Math. Soc. 2005, 11, 33-38.

15. Agarwal, R.P.; O’Regan, D.; Sahu, D.R. Fixed Point Theory for Lipschitzian-Type Mappings with Applications; Topological Fixed Point Theory and Its Applications; Springer: New York, NY, USA, 2009; Volume 6.

16. Karapinar, E.; Noorwali, M. Dragomir and Gosa type inequalities on b-metric spaces. J. Inequal. Appl. 2019, 2019, 29. [CrossRef] 
17. Aydi, H.; Samet, B. On some metric inequalities and applications. J. Funct. Space 2020, 2020, 3842879. [CrossRef]

18. Dragomir, S.S. Refined inequalities for the distance in metric spaces. Prepr. RGMIA Res. Rep. Coll. 2020, $23,119$.

19. Dragomir, S.S. Inequalities for the forward distance in metric spaces. Repr. RGMIA Res. Rep. Coll. 2020, $23,122$.

20. Pečarić, J.E.; Proschan, F.; Tong, Y.L. Convex Functions, Partial Orderings, and Statistical Applications; Mathematics in Science and Engineering; Academic Press: Boston, MA, USA, 1992; Volume 187.

21. Vetro, C.; Vetro, F. Common fixed points of mappings satisfying implicit relations in partial metric spaces. J. Nonlinear Sci. Appl. 2013, 6, 152-161. [CrossRef] 